\title{
Allergic contact dermatitis from transdermal buprenorphine
}

\author{
Kim Vander Hulst ${ }^{1}$, Elisabeth Parera Amer ${ }^{2}$, Claude Jacobs $^{3}$, Virginie Dewulf ${ }^{4}$, Marie Baeck ${ }^{4}$, \\ Ramón M. Pujol Vallverdú ${ }^{2}$, Ana Giménez-Arnau ${ }^{2}$, Dominique Tennstedt ${ }^{4}$ and An Goossens ${ }^{1}$ \\ ${ }^{1}$ Department of Dermatology, University Hospital, K.U. Leuven, B-3000 Leuven, Belgium, ${ }^{2}$ Department of \\ Dermatology, Hospital del Mar, IMAS, Universitat Autònoma, 08003 Barcelona, Spain, ${ }^{3}$ Private Dermatologist, \\ 1150 Brussels, and ${ }^{4}$ Department of Dermatology, University Hospital Saint-Luc, Brussels, 1200 Belgium
}

Background: Buprenorphine is a low-molecular-weight, lipophilic, opioid analgesic. The transdermal delivery system (TDS) containing it has skin irritation potential, but at least two cases of contact allergy to the active principal have been described previously.

Objective: To confirm allergic contact dermatitis from transdermal buprenorphine (TDB) in five older patients suffering from chronic pain and who developed persistent, pruritic erythematous plaques at the contact sites, with two of them also presenting with a generalized skin eruption.

Methods: Besides the baseline patch test series, all five patients were tested with the TDB, four of whom were also tested with the placebo transdermal delivery system as provided by the manufacturer; one patient was also tested with other preparations containing buprenorphine.

Results: All reacted to the TDB containing the active principal, the placebo being negative in the four patients tested. The patient tested with the other buprenorphine preparations did react positively to them as well. Tests with TDB in 28 healthy controls were negative.

Conclusion: We report five cases of delayed hypersensitivity reactions to a TDS containing buprenorphine. Such adverse reactions might be under-reported. A fentanyl-containing TDS is a good alternative in these cases.

Key words: allergic contact dermatitis; alternative; analgesics; buprenorphine; fentanyl; opioids; pain control; transdermal delivery system. (C) Blackwell Munksgaard, 2008.

Accepted for publication 26 July 2008

Buprenorphine is a strong opioid analgesic and is a partial $\mu$-opioid receptor agonist and a semisynthetic derivate of the morphine alkaloid thebaine $(1,2)$. It is available for parenteral, sublingual, and transdermal administration, and since 2001, it has been available in several European countries in a transdermal delivery system (TDS) for the treatment of chronic and moderateto-severe pain of malignant and non-malignant origin (3). Transdermal buprenorphine (TDB) provides a continuous release at $35,52.5$, or $70 \mu \mathrm{g} / \mathrm{hr}$ corresponding to daily doses of 0.84 , 1.26 , and $1.68 \mathrm{mg} /$ day, respectively (2). Buprenorphine is highly lipophilic, has a low molecular weight, and is highly potent, which makes it an ideal medication for transdermal delivery $(1,2,4)$.
The advantages of a TDS are multiple: it is noninvasive, it avoids the first-pass metabolism in the liver, there is a slow and continuous release of the active compound, which leads to a constant and predictable serum drug concentration over a prolonged period, avoiding adverse effects due to plasma peaks, and more frequent dosing; the latter results in better compliance $(1,3,4)$.

\section{Patients and Methods}

\section{Case 1}

A 63-year-old atopic man was referred to the Contact Allergy Unit in Leuven in August 2007, because of intolerance to TDB $(35 \mu \mathrm{g} / \mathrm{hr}$, Transtec ${ }^{\mathbb{R}}$; Grünenthal NV, Sint-Stevens-Woluwe, 
Belgium). He had suffered from chronic muscle and joint pain since the age of 26 years and had been treated with different medications: nonsteroidal anti-inflammatory drugs (acetylsalicylic acid and paracetamol) and the narcotic analgesic tramadol, as advised by the pain clinic. Because of drug adverse events such as headache and gastrointestinal symptoms and lactose intolerance, in September 2004, his physician started treatment with TDB, which resulted in successful pain control. One year later, however, the patient developed pruritic and burning erythema at the contact sites of TDB, especially when sweating. Fluticasone propionate (Flixonase Aqua ${ }^{\mathbb{R}}$; GlaxoSmithkline SA/NV, Genval, Belgium) as well as triamcinolone acetonide $0.1 \%$ in petrolatum (pet.) improved the skin lesions but could not prevent their recurrence. The patient thereupon tried to stop using the patches and to use alternative medication, i.e. tilidine combined with naloxone, but this was insufficient for pain control. He further had a history of intolerance to adhesive plasters.

The patient was tested with the European baseline series (Trolab ${ }^{\mathbb{R}}$; Hermal, Reinbek, Germany), TDB and the placebo transdermal delivery system (PTDS) as provided by the manufacturer, and additional allergens. Positive reactions were observed to TDB (+ D2 and ++ D4) (Fig. 1) and Myroxylon pereirae (balsam of Peru) ( + D4), the latter for which no relevance could be found. Testing with PTDS in this patient and with the TDB in three controls was negative. As an alternative for the Transtec ${ }^{\mathbb{R}}$ patch, fentanyl TDS (Durogesic ${ }^{\mathbb{R}}$; Janssen-Cilag NV, Berchem, Belgium) was started and the patient has since remained pain and lesion-free (contacted by telephone).

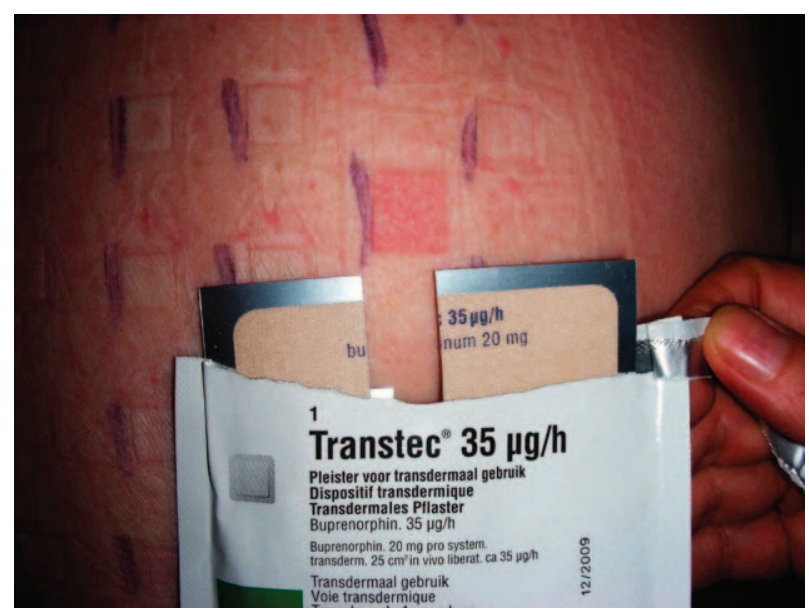

Fig. 1. Positive test $(++\mathrm{D} 2)$ to the Transtec ${ }^{\circledR}$ transdermal delivery patch (transdermal buprenorphine) (case 1).
Case 2

A 79-year-old man with a personal history of penicillin allergy, hypertension, diabetes mellitus, and multiple vertebral fractures secondary to severe osteoporosis was investigated in the Dermatology Department in Hospital del Mar, because of the development of a pruritic, erythematous reaction limited to the site of application of TDB (Transtec ${ }^{\mathbb{R}}$; Grünenthal, Madrid, Spain). He had been applying TDB for several weeks for chronic back pain before the lesions developed. After TDB withdrawal and topical high-potency corticosteroids, the lesions resolved. Patch tests with TDB showed a papular erythematous reaction $(++$ D4), suggesting a delayed hypersensitivity reaction. The baseline patch test series $\left(\right.$ Trolab $^{\mathbb{R}}$; Almirall, Reinbek, Germany) and the adhesive part of TDB were negative. No further testing could be performed because the patient died.

\section{Case 3}

A 90-year-old woman with a history of hypertension, diabetes mellitus, and duodenal ulcer was referred to the same department as in case 2. During the previous year, she had at least four episodes of erythema and transient oedema on the face and eczematous lesions on the eyelids. These signs persisted for more than 1 day and were considered to be consistent with a delayed hypersensitivity reaction. She also reported episodes of bronchospasm. She was using TDB (Transtec ${ }^{\mathbb{R}}$ ) because of chronic pain secondary to a degenerative arthropathy as well as several other drugs, i.e. losartan, furosemide, pentoxifiline, naproxene, omeprazole, and miglitol. There was also a weak erythematous and persistent infiltrated plaque at the application site of the TDB. After withdrawal of buprenorphine, furosemide, naproxene, and pentoxifiline, the symptoms disappeared. Patch tests were performed and positive reactions were observed to TDB (as is) $(++\mathrm{D} 4)$ and to three forms of Buprex ${ }^{\circledR}$ (Schering-Plough, Madrid, Spain) (++ D4), i.e. the i.v. solution containing buprenorphine $0.3 \%$ aqueous, the sublingual tablet Buprex $0.2 \mathrm{mg}$ (in powder as is), and the same tablet (in powder and moistened with water) (Fig. 2). In the baseline patch test series, $M$. pereirae (balsam of Peru) (++ D4) and neomycin $(++$ D4) were positive. PTDS as provided by Grünenthal, the non-steroidal anti-inflammatory drug series (Martí Tor, Barcelona, Spain) and losartan (a 12.5-mg tablet in powder and diluted in a minimum amount of pet.) showed negative results. All drugs, except buprenorphine, were successively reintroduced without adverse reactions. The relation between the bronchospasm 


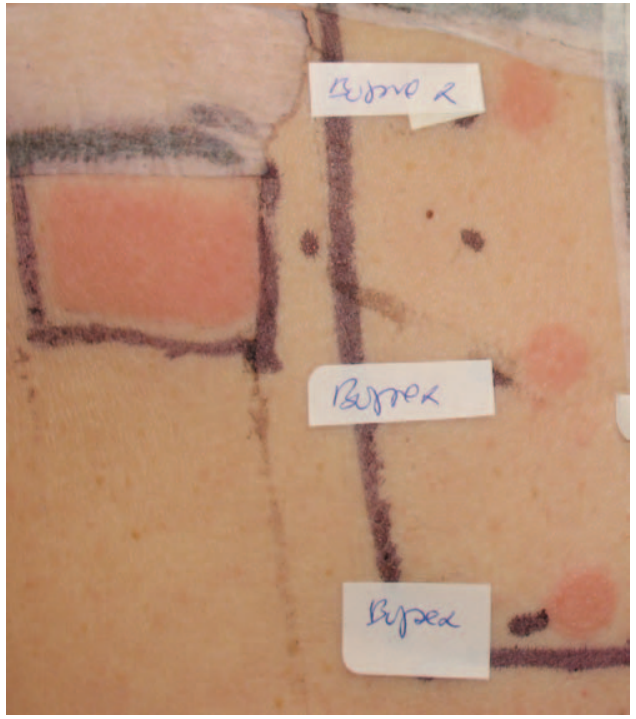

Fig. 2. Positive tests $\left(++\right.$ D4) to the Transtec ${ }^{\mathbb{R}}$ transdermal buprenorphine and to several Buprex ${ }^{\mathbb{R}}$ preparations (case 3).

and the TDB was not clear, but the symptoms did disappear after its withdrawal. The dermatitis was considered to be both a local and a systemic allergic reaction to buprenorphine. Tests with TDB in 25 healthy controls were negative.

\section{Case 4}

A 72-year-old man with a history of chronic urticaria and intestinal disease was observed by a dermatologist (C. J.) in Brussels. He had been applying Transtec $^{\mathbb{R}}$ TDS patches because of skeletal pain but after a few applications had started to develop eczematous reactions under the central zone of the patches. The patient was tested with the baseline and acrylate series (Chemotechnique, Vellinge, Sweden), TDB, and PTDS, but he only reacted to the patch containing the active principal $(++\mathrm{D} 2$ and +++ D4). The patient had satisfactory pain control with systemic tramadol hydrochloride.

\section{Case 5}

A 79-year-old woman was admitted to the Department of Dermatology of the University hospital Saint-Luc in Brussels in March 2008, for a recurrent, generalized eczematous eruption present for 1 year. The first episode had followed a toxicoderma due to clarithromycin and lowmolecular-weight heparins. The eczematous eruptions had been treated with potent topical corticosteroids (betamethasone dipropionate and clobetasol propionate) and sometimes with systemic corticosteroids. She had known contact allergies: patch tests performed in 1986 and 2007 had shown positive reactions to potassium dichromate, nickel sulfate, $p$-phenylenediamine, fragrance mix 1 , and ethylenediamine. She had used fentanyl TDS (Durogesic ${ }^{\mathbb{R}}$ ) because of back pain due to scoliosis, which was stopped because of confusion problems. Transtec ${ }^{\circledR}$ TDS patches were applied from February 2008, but the patient developed itchy plaques at the contact sites. Patch tests were performed with the European baseline standard series and her medication, including Transtec ${ }^{\mathbb{R}}$ TDS and PTDS and Durogesic ${ }^{\mathbb{R}}$ TDS. Positive patch test reactions were observed to budesonide ( + D4), ethylenediamine $(++$ D4), tixocortol pivalate $\left(+\right.$ D4), and Transtec ${ }^{\mathbb{R}}$ TDS $(+$ D4). Durogesic ${ }^{\mathbb{R}}$ TDS and PTDS remained negative. The patient's recurrent generalized eczema possibly had different causes: atopic constitution and contact allergy to corticosteroids, but more probably a local and systemic allergic reaction to buprenorphine. Indeed, the patient had not used any topical corticosteroid before the exacerbation of the skin eruption, and the lesions considerably improved after withdrawal of TDB. The patient was put on systemic tramadol hydrochloride, which provided satisfactory pain control.

\section{Results}

The diagnosis in the five cases, three men and two women, was a delayed hypersensitivity reaction to the TDS containing buprenorphine $\left(\right.$ Transtec $^{\mathbb{R}}$ ) used to treat chronic pain. Two patients also presented with systemic allergic dermatitis. Table 1 gives the patch test results obtained. Tests with TDB in 28 controls were negative.

\section{Discussion}

Allergic contact dermatitis from TDB has not often been described. We found two case reports in the literature, both from Spain $(5,6)$. In the latter, the authors also reported on another case, which they considered to be irritant (7). In a postmarketing surveillance study of TDB in 13179 patients, Griessinger et al. reported 109 contact dermatitis cases $(0.8 \%)$. They did not specify whether the dermatitis was due to the adhesive or active compound. In the same study, they also reported pruritus and an erythematous rash as an adverse drug reaction in $0.7 \%$ and $0.5 \%$ of the patients, respectively (3). During the clinical development programme of the buprenorphine TDS, which consisted of three double-blind, parallel group, placebo-controlled studies and one open-label follow-up study, erythema was seen in $25.4 \%$ and pruritus in $22 \%$ of all patients. All local adverse events were considered mild to moderate in intensity (4). 
Table 1. Positive patch test reactions in the five subjects reacting to buprenorphine TDS (Transtec ${ }^{\circledR}$ )

\begin{tabular}{|c|c|c|c|c|c|c|c|}
\hline & Sex & $\begin{array}{l}\text { Age } \\
\text { (years) }\end{array}$ & $\begin{array}{l}\text { Duration of } \\
\text { application }\end{array}$ & $\begin{array}{l}\text { Transtec }^{\circledR} \\
\text { TDS }\end{array}$ & $\begin{array}{l}\text { Placebo } \\
\text { TDS }\end{array}$ & Other positive tests & Alternative \\
\hline Case 1 & M & 63 & 1 year & + & - & Myroxylon pereirae & Fentanyl \\
\hline Case 2 & M & 79 & Several weeks & + & NT & - & Patient died \\
\hline Case 3 & $\mathrm{~F}$ & 90 & 1 year & + & - & $\begin{array}{l}\text { Buprenorphine } \\
M . \text { pereirae neomycin }\end{array}$ & Fentanyl \\
\hline Case 4 & M & 72 & A few applications & + & - & - & Tramadol hydrochloride \\
\hline Case 5 & $\mathrm{~F}$ & 79 & $<2$ months & + & - & $\begin{array}{l}\text { Potassium dichromate, } \\
\text { nickel sulfate, } \\
\text { p-phenylenediamine, } \\
\text { ethylenediamine, fragrance mix } 1, \\
\text { budesonide, tixocortol pivalate }\end{array}$ & Tramadol hydrochloride \\
\hline
\end{tabular}

F, female; M, male; NT, not tested; TDS, transdermal delivery system.

Besides local reactions at the application site, a hypersensitivity reaction to buprenorphine may also become generalized or produce a systemic reaction, such as in our cases 3 and 5. The transdermal system, being a systemic way of drug delivery, may be more prone to induce such reactions.

In agreement with the literature (6), if a transdermal system is to be preferred, fentanyl TDS is a good alternative, as it is also a strong opioid analgesic. It differs from buprenorphine because of its full $\mu$-opioid receptor agonist character and its chemical structure, namely it is a member of the phenyl piperidine class of opioids (1). According to a small study comparing both TDS systems, it was found that local adverse events ('skin irritation' reactions) were more often seen with transdermal buprenorphine than with transdermal fentanyl (8).

Other opioids, such as codeine, morphine, and heroine, have been reported as causes of occupational contact dermatitis in pharmaceutical workers and health care units (9-11). These drugs may also be responsible for generalized dermatitis after systemic administration $(9,12,13)$, for which positive patch tests to the opioids suggested a type IV immunological mechanism. Moreover, crossreactions between structural related opioids have been described $(9,12)$.

\section{Conclusion}

We report five cases of delayed hypersensitivity reactions to a TDS containing buprenorphine. All patients developed pruritic erythematous plaques at the contact sites and two also suffered from generalized dermatitis. When a transdermal system is preferred, the TDS containing fentanyl has shown to be a good alternative to control severe pain.

\section{Acknowledgement}

We are very grateful to Grünenthal NV from Belgium and Spain for providing us with the placebo Transtec ${ }^{\mathbb{R}}$ TDS for epicutaneous testing.

\section{References}

1. Zuurmond W W, Meert T F, Noorduin H. Partial versus full agonists for opioid-mediated analgesia - focus on fentalyl and buprenorphine. Acta Anaesthesiologica Belgica 2002: 53: 193-201.

2. Johnson R E, Fudala P J, Payne R. Buprenorphine: consideration for pain management. J Pain Symptom Manage 2005: 29: 297-326.

3. Griessinger N, Sittl R, Likar R. Transdermal buprenorphine in clinical practice - a post-marketing surveillance study in 13179 patients. Curr Med Res Opin 2005: 21: 1147-1156.

4. Böhme K. Buprenorphine in a transdermal therapeutic system - a new option. Clini Rheumatol 2002: 21 (Suppl. 1): S13-S16.

5. Callejo Melgosa A M, Martínez J C, Fuentes M J, Martín M. Allergic contact dermatitis from buprenorphine. Allergy 2005 : 60: $1217-1218$.

6. Pérez-Pérez L, Cabanillas M, Loureiro M, FrenándezRedondo V, Labandeira J, Toribio J. Allergic contact dermatitis due to transdermal buprenorphine. Contact Dermatitis 2008: 58: 310-312.

7. Fernández-Redondo V, Concheiro J, Pérez-Pérez L, Toribio J. Contact dermatitis and transdermal buprenorphine. Contact Dermatitis 2008: 58 (Suppl. 1): 74.

8. Schmid-Grendelmeier P, Pokorny R, Gasser U E, Richarz U. A comparison of the skin irritation potential of transdermal fentanyl versus transdermal buprenorphine in middle-aged to elderly healthy volunteers. Curr Med Res Opin 2006: 22: 501-509.

9. Hogen Esch A J, Vander heide S, Van den brink W, Van ree J M, Bruynzeel D P, Coenraads P J. Contact allergy and respiratory/mucosal complaints from heroin (diacetylmorphine). Contact Dermatitis 2006: 54: 42-49.

10. Waclawski E R, Aldridge R. Occupational dermatitis from thebaine and codeine. Contact Dermatitis 1995: 33: 51.

11. Condé-Salazar L, Guimaraens D, Gonzáles M, Fuente C. Occupational allergic contact dermatitis from opium alkaloids. Contact Dermatitis 1991: 25: 202.

12. Rodríguez A, Barranco R, Latasa M, Ortiz de Urbina J J, Estrada J L. Generalized dermatitis due to codeine. Crosssensitization among opium alkaloids. Contact Dermatitis 2005: 53: 240 .

13. De Cuyper C, Goeteyn M. Systemic contact dermatitis from subcutaneous hydromorphone. Contact Dermatitis 1992: 27: 220-223.

Address:

An Goossens

Department of Dermatology

University Hospital

K.U. Leuven

B-3000 Leuven, Belgium

Tel: 003216337860

Fax: 003216337012

e-mail:an.goossens@uz.kuleuven.ac.be 